\title{
16
}

\section{The conference/classroom of the future: an interdisciplinary approach}

\author{
M. Mühlhäuser, J. Borchers, C. Falkowski, K. Manske \\ Telecooperation Research Group, Dpt. of Computer Science, Linz University \\ Altenberger Str. 69, 4040 Linz, Austria \\ Phone: ++43-732-2468-9239, Fax: ++43-732-2468-10 \\ E-mail: $\{$ max,jan,chris,knut $\}$ atk.uni-linz.ac.at \\ WWW: http://www.tk.uni-linz.ac.at/
}

\begin{abstract}
A vital step towards the vision of an 'international office' is to create actual environments in which different design options and solution strategies can be evaluated in practice. This paper describes the Conference/Classroom of the Future (CCF) project which aims at setting up a learning and working environment that uses advanced information technology to support cooperative activities. The CCF project takes an integrated approach to address aspects ranging from interior design and information technology to software support and work organization.

From a characterization of learning and working in the next century, we develop usage scenarios, and explain our design decisions concerning furniture, hardware, operating systems, and especially the necessary software infrastructure to support cooperation. Our initial 'visions' are described as well as what we found was achievable under real-world financial and technological constraints. We also outline what software support is still missing for such an environment, and our plans to provide this support in the course of the project. We conclude with a realistic 'shopping list' for people who have to manage similar projects.
\end{abstract}

\section{Keywords}

Classroom, learning environments, conference room, design, CSCW, telecommunications, telemedia, teleteaching, cooperative systems, IT platform requirements, IS infrastructure 


\section{INTRODUCTION}

\subsection{Motivation}

The rather ambitious idea of an 'international office of the future' requires that different strategies and approaches are not just being discussed, but implemented and evaluated under real-world conditions. In the Conference/Classroom of the Future (CCF) project, our research group is currently creating such a sample environment. While the CCF project is still under development, the design phase has been completed.

The authors are responsible for the definition of concepts ('visions') for this area, and for the delivery of suitable exhibits that put those concepts into practice ('reality'). Due to these two different tasks, we have tried where possible to compare our 'visions' for certain aspects with the 'reality' we have been able to achieve with given current state-of-the-art tools and our budget constraints. We hope that this form of presentation will be helpful to those who are planning or designing similar environments.

\subsection{Overview}

The remainder of this paper is organized as follows:

Section 2 introduces the project environment in which the CCF is embedded, the Ars Electronica Center (AEC) and its Telemedia/Teleteaching floor. We briefly sketch the overall goals, components, and project status of this organizational framework, as far as they are relevant to the CCF.

Given that background information, we turn to design: Section 3 presents how we expect learning and working processes to change in the future, and how this is reflected in our usage assumptions and conceptual design strategies. The 'overall message' that we will try to convey to visitors and users of the CCF is presented here.

Starting with section 4, we describe our design decisions in a bottom-up fashion: First, we summarize our ideas regarding CCF furniture, i.e., which desk configurations are required, and how this effects the design of individual computer desks.

Section 5 then addresses base technology questions like the choice of workstations, displays, portable devices, network technology, and periphery.

Our decision for a certain operating system with its advantages and disadvantages is explained in section 6.

Section 7 deals with the software infrastructure required for meaningful cooperation across application and computer boundaries. Since this is still a research-intensive area, we have focused our own development efforts on this problem, and describe them in more detail in this section. Problems addressed here include cooperation control applications, mobility and workflow support, hypermedia access beyond WWW, telepresence, electronic books, low-bandwidth multimedia collaboration, and semantic meeting assistance.

On top of this infrastructure, applications and curricular contents have to be added to the CCF scenario. Section 8 addresses this issue.

Section 9, the conclusion, summarizes what we think can be learned from this project already, and, for the practitioner, gives a list of hardware and software components we recommend as equipment when designing similar environments. 


\section{BACKGROUND}

This section outlines the background of the CCF project, the AEC and its Telemedia/Teleteaching floor.

\subsection{The Ars Electronica Center (AEC)}

The AEC is an exhibition and activity venue centered around the question how new information technology will influence the way we will live, work, communicate, and relax in the next century. The AEC project developed out of the annual Ars Electronica festival, one of the most influential computer arts festivals in the world since 1979. The AEC is currently being designed and built in the very heart of the city of Linz, the capital of Upper Austria. This part of Austria belongs to the 'motors of Europe', a number of regions selected by the EU as pace-makers on the way to the common european market due to their economic strength and innovative potential. The AEC will open in September 1996, and will consist of five floors addressing different aspects of innovative information technology, its applications and implications, like virtual realities, the Internet, etc.

\subsection{Telemedia/Teleteaching floor}

A major part of the AEC is the Telemedia/Teleteaching floor where special emphasis is laid on the future of learning and working. It will consist of the following areas which are described below:

- the Conference/Classroom of the Future (CCF);

- an 'extended living-room';

- an exhibition area.

The CCF is a thoroughly designed environment for cooperative learning and working. The remaining paper will focus on this area because it is the most important project for an 'international office of the future'. Although the remaining two areas are no less interesting, they can only be presented briefly here.

The 'extended living-room' is a leisurely equipped area contrasting the 'ergonomically correct' office equipment of the CCF. It puts Mark Weiser's vision of such an area (Weiser, 1991) to a test, offering a group of comfortable armchairs and sofas where people can relax in a comfortable environment and still use mobile computing devices to access network resources. We hope that this relaxed atmosphere will help overcome hierarchical, social, and cultural barriers, in the same way as the most fruitful discussions at work often take place informally, during a break or at similar occasions where the standard office environment is left.

The exhibition area will consist of a number of individual interactive exhibits, or 'actibits', which are intended to give insight into new ways of learning and working, as affected by upcoming networked multimedia and computing technology. The planned installations comprise

- New Media: demonstrating the pitfalls and potential of interactive TV (ITV), and the role of the Internet among the publication media (includes Internet guided tours);

- New Learning: e.g., presenting novel approaches to understanding musical concepts and composing and playing music, optionally together with remote players over the net (the WorldBeat project); 


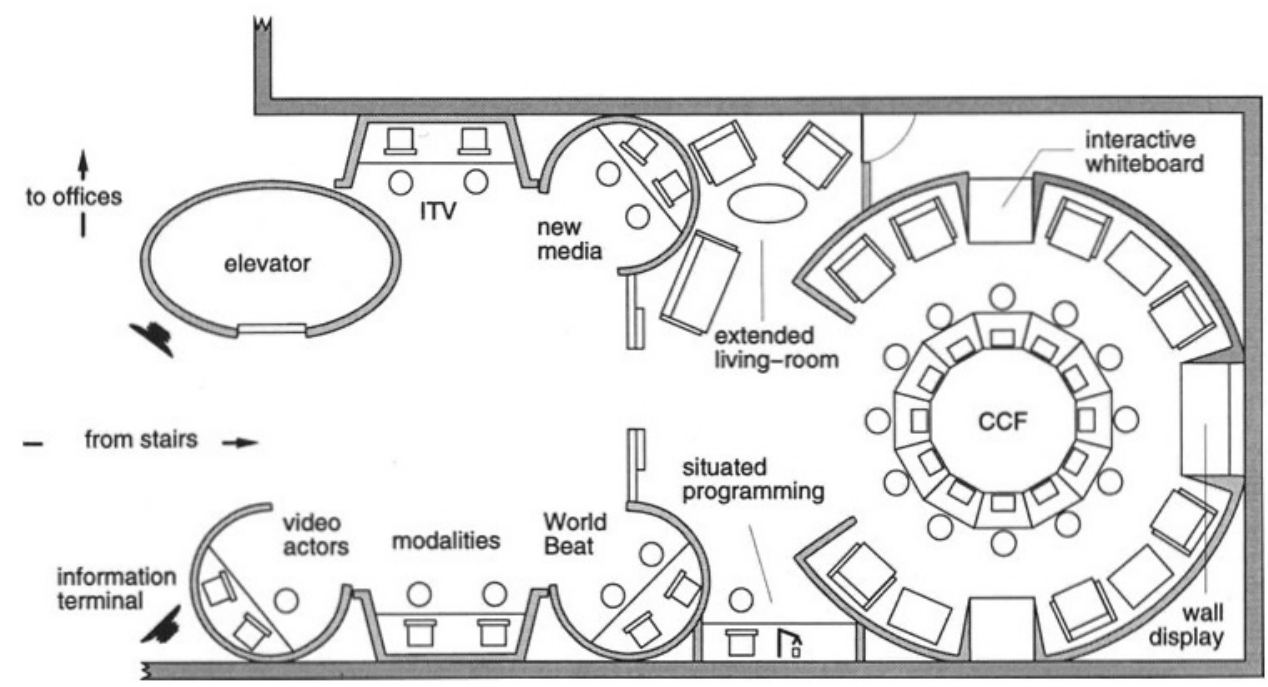

Figure 1 Overview of the Telemedia/Teleteaching floor.

- New Interfaces: investigating the use of new input methods like hand-gesture recognition, and new user interface metaphors like video actors ('avatars');

- Situated Programming: gaining programming experience with immediate feedback, based on robot programming with a simple language.

Figure 1 gives an overview of the Telemedia/Teleteaching floor. Note that for administrative and security reasons, the 'Situated Programming' installation is co-located with the CCF.

\section{CONCEPTUAL DESIGN}

This section presents the 'messages' we had in mind when we started to think about CCF design, and our usage concept for the environment.

\subsection{Integration of learning and working}

As the term 'Conference/Classroom' indicates, we envision that the classical boundaries between scholarship and worklife, between classroom and cooperative office space, may and should become blurred in the future. Cooperative learning and cooperative work tend to resemble each other more and more, so that future classrooms will have to look more like conference or meeting rooms. We will give two main reasons for this assumption here:

1. Traditional one-to-many teaching scenarios are outdated in a world in which facts and even concepts become outdated too fast for any teacher to keep pace with. Moreover, cooperation and information acquisition skills, rather than individual facts or concepts, become the very subject of 
learning. As a result of these trends, future classrooms should support teacher-mediated cooperative information acquisition in which learners play an active role and mutually teach each other.

2. At the same time, it is well-known that learning is becoming a life-long task. Proper information acquisition and processing becomes a major productivity and success factor in organizations worldwide. Organizations are becoming regionally dispersed, i.e., physically distributed, even on a world-wide scale. Cooperative work in locally and remotely distributed systems becomes crucial in such an environment, as does networked access to worldwide information resources.

After all, the idea of a conference is to have a meeting, usually moderated by one person, where people learn from each other, develop new ideas together, and finish with a new status and new tasks for their project - and doesn't that sound just like the ideal form of a school lesson as well?

Finally, information propagation becomes as important for an organization as information acquisition. Thus, the Class/Conference Room of the Future has to facilitate proper generation of networked, multimedia information as well as its retrieval and consumption.

As a consequence, we label this combination of learning and working in the future as 'cooperative information processing', or simply 'learning'. In the remainder of this paper, we ask the reader to understand the term 'learning' in this broader sense.

\subsection{Work focus objects}

The fact that learning and working scenarios converge had to be accomodated by our design. Conference and classroom environments as they are known today, however, show very divergent design and setup characteristics. Looking for common ground between the two environments, we isolated a concept which we labelled 'work focus object':

At both sorts of activities, whether conference- or tutorial-like, attention is usually focused on a central object. This can be a report to be discussed in a meeting, a paper to be written in a joint editing session, or a tutor and the material he presents in a seminar. Thus, a work focus object in the general case is the object which the cooperative information processing activity uses to represent its current state and history. As everybody knows from meetings, such an object is essential, even if it is just to ensure that everybody is talking about the same thing.

Our idea of a work focus object influenced subsequent design decisions. For example, it needed a medium to represent it, which we provided through interactive collaborative whiteboards for group work, and through a large wall-mounted display for tutorials.

\subsection{Three major aspects of future learning/working}

With learning and working scenarios merged and centered around work focus objects, we continued our design of a CCF concept by investigating which major changes new technology will be able to bring to this way of information processing. We identified three major attributes that we think will characterize 'learning' in its broader sense in the next century, and that can be improved through the use of cooperative hypermedia concepts and technology:

1. situated: There will be a tendency to embed learning into a real situation ('learning by doing'). This may be achieved in three different ways:

- simulated: The computer simulates technical processes; by controlling or programming these 'digital worlds' (e.g., virtual realities), the learner understands their underlying concepts. 
- analog: The computer serves merely as a tool to organize work in a 'real-world' project; it serves as an amplifier of, not as a barrier from, the actual project contents (Kay, 1991).

- digital: The things to be learned are actually taking place inside the computer, or the network; by joining this process the learner understands its nature (example: internet commerce).

2. associative: Structure, contents, and participants of a working or learning process are considered part of a network:

- interdisciplinary: Material is presented as 'seamless' hypermedia instead of being limited by classical subject/faculty borders and linear structure.

- cooperative: It is understood that in the future people and organizations will only be able to work together in a cooperative environment, emphasizing team work aspects.

- networked: The technical infrastructure of world-wide (mobile) communication networks will grow to support this style of learning and working.

3. motivating: Making learning fun remains the biggest challenge. Computer support can make learning more:

- individual: Personal preferences (way of presentation, specific interests, etc.) can be taken into account.

- didactic: New so-called instructional strategies can draw from the unique advantages of computer use for making cooperative information processing more interesting (example: group adventure framework to convey a certain subject).

- attractive: Presentation of learning material that makes reasonable use of hypertext concepts and multimedia technology can make teaching come alive.

\subsection{Usage scenarios}

Having defined which aspects of future learning we wished to emphasize, we needed to understand how the room was to be used before we could make decisions about its interior design and equipment. After all, we wanted this room to be much more than just an exhibition area where casual visitors pass by, read some of our 'visions' on cardboard posters (or on-screen presentations, for that matter), play with some neat innovative input device, and walk on - we wanted the infrastructure to be usable by actual meetings, industrial seminars, classroom lessons, or any other kind of cooperative activity that could take advantage of the technology available. Of course, the casual visitor would still represent a major part of the people visiting the CCF, so we could not neglect this target group.

At this stage the decision to merge conference and classroom concepts - as elegant as it may be posed a number of complex design problems. As Shneiderman (1995) points out, workplaces have to be flexible to accomodate different configurations that cater for a variety of usage scenarios.

Therefore, we decided on the following assumptions regarding CCF usage:

1. 'Cooperative work' comprises both learning scenarios ('Tutorials' with 1 teacher/tutor and $n$ learners) and conferencing scenarios (with $n$ participants and possibly 1 moderator).

2. Target groups of the CCF are

- individual, casual visitors;

- school classes; 
- adult education courses;

- internal seminars carried out by the AEC;

- external, industrial seminars.

3. The room is always in one of two states: used (e.g., for tutorials, conferences, etc.), or unused (when only individuals are accessing the workstations).

4. Cooperative work is always centered around 'work focus objects' that constitute the focus of attention. They may be a person (e.g., a teacher, tutor, or moderator), and/or a document (blackboard, overhead presentation slides, lecture notes, handout, etc.)

5. Cooperative projects are usually carried out in a sequence of two alternating phases:

- individual contribution by means of personal computer access;

- cooperative verification, using a common medium (like a blackboard) to represent the work focus object.

6. According to their size, events taking place in the CCF can be classified into five scenarios:

(a) individual use by single interested visitors who wish to try out the technology available (the only configuration where the room is considered unused);

(b) one or two small groups of up to four people each, working in a cooperative, meeting-like manner, and using CCF technology to talk about a common subject;

(c) Seminar of up to twelve people, where the focus of attention is a tutor/teacher presenting some information on a large display;

(d) Conference of four to twelve people, using a U-shaped desk configuration that is virtually extendable via a large, wall-mounted display (see section 7);

(e) Class of 12 to 24 people, using a large U-shaped, or a circular configuration of workplaces which are usually shared by two learners.

\section{FURNITURE CONSIDERATIONS}

The above descriptions showed clearly that a very versatile furniture concept was needed. We met the requirements by choosing a collection of twelve separate desks. These desks were designed with a trapezium-shaped desktop at an angle that allows a circle configuration. They are accompanied by optional triangular connecting pieces (at $30^{\circ}$ ) to create linear configurations like the ends of a U-shaped conference desk.

While Figures 1 and 2 show setups with one seat per workstation, the desks are large enough for two people to work in front of each computer. This setup is recommended by many experts in the field of computer-aided learning - see, for example, Shneiderman (1995) -, especially since it encourages discussion.

By replacing half of the desks with standard tables, a more spacious setup can be created which is essential for some curricular activities where a lot of additional learning material (e.g., robotics construction kits), has to be accessible at the workplaces. For very large groups, four additional tables can be added, resulting in 16 tables altogether, for example in an oval instead of the circular arrangement.

The interior design of the CCF was subcontracted to an architect with experience in exposition design, and is not within the scope of this paper. 


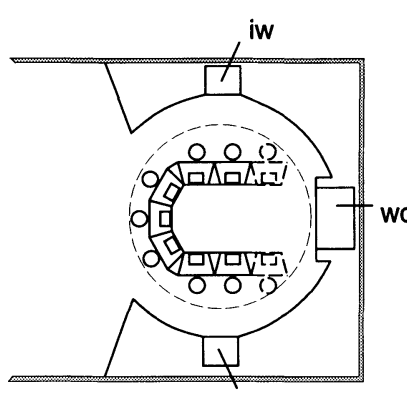

iw

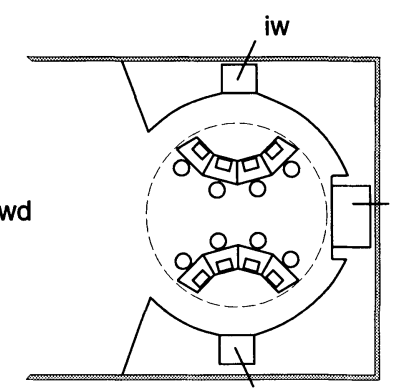

iw

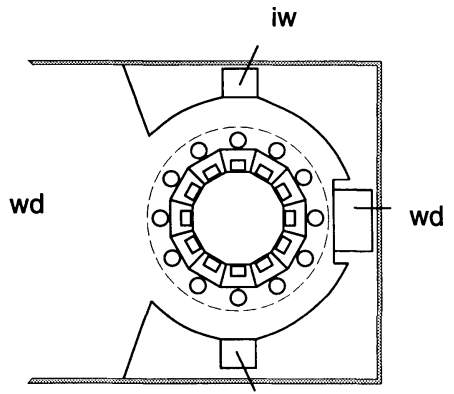

iw

Figure 2 The three alternative desk configurations within the CCF: conference, small groups, and circular setup (iw: interactive whiteboard, wd: wall-mounted display).

\section{BASE TECHNOLOGY}

\subsection{Workstations}

For individually oriented subtasks, we decided to equip the CCF with twelve networked workstations, usable by one to two people each. This number was chosen not only due to available space, but also has didactic reasons: Twelve workstations divide nicely into a number of smaller groups, and groups larger than 24 persons make cooperative work increasingly complex and hard to manage. (Four additional desks workstations are available for extraordinarily large groups.)

Our vision was to put into reality the idea of ubiquitous computing (Hayter, 1993): The computer becomes 'invisible' and unobtrusive, getting 'out of the way of work'. The specific device becomes exchangeable because the network allows for automatic migration of personal data and applications to whatever device the user picks up.

This was where a basic conflict first became visible: while ambitious projects such as 'ubiquitous computing' are intriguing from a theoretical point of view, the hardware and software for such projects is usually not available for general use yet. Furthermore, most commercial application development takes place for industry-standard machines and operating systems, which means that a variety of software is available at a much lower price for those environments. This is a serious issue when budget constraints are to be considered.

In addition, a learning environment such as the CCF has to take into account that it is supposed to be usable by companies, schools, etc. Teachers, for example, will want to use their Powerpoint slides without having to create them from scratch or go through complicated conversion routines. This shows that projects like the CCF are always a compromise between innovation and compatibility with existing standards.

Unix workstations, for example, would have been advantageous for a number of reasons: their operating and file system architecture inherently supports multiple users and preemptive multitasking, which makes them much more robust, secure, and less prone to complete system crashes. Also, their widespread use in the research domain makes them attractive since it means that many prototype implementations first become available on these machines.

We also considered Macintosh machines, which would have been preferable due to their more ho- 
mogeneous system architecture, more intuitive user interface, and clean support of extensions like multiple monitors for a single desktop, audio/video applications (through QuickTime), etc.

In our case, however, software availability, price levels, and our cooperative talks with hardware producers showed that a PC-based solution would be the best choice. We finally agreed with SiemensNixdorf Information Systems (SNI) to install their Scenic Multimedia PCs in the CCF. These machines are from SNI's latest series of personal computers, based on a $100 \mathrm{MHz}$ Pentium board with PCI bus technology, and come with 16 MB RAM, a 1 GB hard disk, a quad-speed CD-ROM drive, sound card, fax modem, and a Windows '95 licence. We will add MPEG compliant video hardware to all machines. We aim at keeping the configuration of those machines as homogeneous as possible to minimize maintenance problems.

Nevertheless, we decided to incorporate at least one Unix machine (as file server to reduce data redundancy), and one Macintosh computer (for the music installation and as a multimedia production station) into the Telemedia/Teleteaching area, to ensure that we could also run future software for those systems, in case it is of interest to us as demonstration or educational content.

\subsection{Displays}

LCD panels would be preferable as display technology because they have a flat, non-distorting surface, are flicker-free, and non-emmissive, putting less strain on the eyes. Nevertheless, we equipped each workplace with a colour CRT monitor, as it is not only substantially less expensive, but also still offers higher resolution and faster response (moving objects do not leave shadows behind). Those aspects are especially important for animation and live video transmission.

To move the rather large monitors 'out of the way' we chose to lower them into the desktop so that people can look across them to see each other. Since our goal was to support social interaction through the computer instead of preventing it, this design detail was important to avoid blocking human-human communication.

\subsection{Interactive group devices}

To support cooperative activity, we found that interactive whiteboards (touch-sensitive boards with computer-generated rear projection, in our case those from Smart Technologies, Inc.) were most suitable.

In the 'small groups' scenario described above, they serve as work focus objects by displaying and representing the status of a discussion, brainstorming, or other cooperative activity. In the circular and conference scenarios, the two interactive whiteboards are used in a new kind of setup. They are fully synchronized, so that participants can easily look at the display of the work focus object at the opposite wall without having to turn their head. For active contribution, users can either access the computers on their desks, or use the whiteboard closest to their seat (which will usually be different from the one they look at).

We also included a large, modular, LCD back-projection based electronic projection wall for presentations and the virtual conference desk extension. Its initial size will be $2 \times 2$ modules, i.e., approx. $1.8 \times 1.5 \mathrm{~m}$. It is scalable, so that it can be extended later as budget allows. This device can be accessed as a single screen, and will be particularly useful in the 'Telepresence' context discussed in section 7 . 


\subsection{Portable devices}

We wanted to add portable computing devices to part of the workplaces, for note-taking as well as to take to the extended living-room. Our options were to choose a PDA, notebook, or mobile i/o device. Most PDAs do not offer sufficient display resolution yet. This is less of a problem with notebooks, but they are often too heavy to be used comfortably, especially in the extended living-room. Moreover, they mean another computer per workplace, which results in additional maintenance load and possible system compatibility problems.

Because of this, we also considered installing Zenith Cruisepads in the CCF. These portable LCD panels are touch-sensitive and work as combined screen and input device for the computer to which they are connected wirelessly. This design results in less weight and battery usage, important points for everyday use in a public space. However, while our vision had been to use such devices with highresolution, color displays, reality showed that Cruisepads and similar devices are not available yet in higher than standard VGA resolutions, mostly black and white, and also seem too slow for graphical aplications. Our final decision for a certain technology therefore still has to be made.

\subsection{Network and periphery}

With telecooperation as a central aspect of our visions, state-of-the-art network infrastructure was compulsory. In this case, we managed to make reality come up to our expectations. The whole AEC will be equipped with three types of network connections, Ethernet, FDDI, and ATM. Since, at least today, the applicability of ATM technology is still very limited, and the Ethernet connections will offer a capacity of $100 \mathrm{Mbit} / \mathrm{s}$ switched, we decided to postpone ATM boards unitl they become necessary for future applications. Within the CCF, combined power/network outlets will be integrated into the floor at a number of places so that desks can always be easily connected to an outlet nearby, for all configurations mentioned above.

An important issue, especially with occasional visitors who do not have adequate hardware at home, is that the interface to and from printed media is of high quality and easy to use. To accomplish this, we added a colour scanner and two laser printers (one colour, one black-and-white) to our equipment list.

We also had to address the question of peripheral devices. Should camera, disk drive, or CD-ROM drive be made available at each of the workplaces? Due to public access, we decided not to make any local drives available to the user, also because of the danger of copyright infringement. Floppy disks and CD-ROMs will be directly accessible on a dedicated and supervised machine instead, or they may be inserted there and accessed simultaneously by several people in the CCF (useful for CD-ROMs). CD sharing software can help overcoming problems of concurrent access by multiple users.

A camera, however, is something we believe belongs in every workplace, especially as it is required for video-conferencing scenarios.

\section{OPERATING SYSTEMS}

Our vision for the CCF was to use a single operating system that supported migrating windows and applications, that included system-level support for collaborative applications (like document sharing, joint editing, etc.), and that was widely available together with standard applications.

Reality was different, however: Magic Cap, for example, would be an interesting choice due to its extensions beyond the desktop metaphor, but most specialized hardware (like the interactive white- 
boards, for example) comes with drivers and dedicated applications for some MS Windows variant only. Moreover, since all computers are to be supervised by a central control station, each additional operating system meant that an interface to this control system would have to be implemented. To keep a minimum of multi-user, security, and multitasking capabilities, we decided to use Windows NT as predominant operating system. We created a boot-time option to run Windows 3.11 or 95 in case an application does not work under NT's compatibility modes as expected. Some Mac and Unix systems will be available as well, as indicated in our machine decisions in section 5, which, of course, cannot really be separated from an OS decision.

\section{SOFTWARE INFRASTRUCTURE AND APPLICATIONS}

For the reasons described in the last section, we focused on developing a suitable software infrastructure for Windows NT platforms.

Our primary task in the AEC Telemedia/Teleteaching project was to coordinate its design and equipment. This meant that we had three choices for the various subtasks:

- find appropriate third-party applications, academic or commercial, and agree with their authors upon terms for incorporation into the CCF environment;

- find external developers who create certain software on our behalf;

- develop software ourselves within our research areas.

Depending on the nature and size of the different projects we chose one of the above options for each of the subprojects we agreed upon. These projects, which are being worked on at the time of writing (February '96), are described in the following sections.

\subsection{Process control and remote operation}

To keep the maintenance of CCF workstations manageable, a system is being developed that will provide access to the processes running on all systems, allowing easy surveillance of tasks, and error recovery support.

While this system will be used to do local maintenance, we are also planning to include a remote control option for CCF workstations so that we can access the machines from our research facilities for testing purposes. In a limited form, this functionality will also be useful for curricular use: A teacher, for example, will be able to directly access a student's computer to help him with problems he has with an application. A number of software packages already exists for this task, including Symantec's 'Norton pcANYWHERE for Windows', Ocean Isle's 'ReachOut Remote Control', and 'Remote Teach/ Remote Desktop' developed at Linz University.

\subsection{Integrated cooperation services}

On of the most important features of the CCF from a user perspective will be cooperation-awareness, i.e., the possibility to use CCF facilities for group activites. Our goal is to provide services for joint editing, shared whiteboard access, and other forms of application sharing that can be used even with standard applications that are not cooperation-aware themselves.

To resolve concurrent access conflicts, we decided that two models of joint control should be supplied: 
1. alternating input device control - only one user controls the input activities on a device at any time, and device control is managed through a 'token' mechanism and/or assigning different 'roles' (e.g., teacher/student) to the users;

2. cooperative input device control - several users concurrently use the input device; e.g., movement of the mouse pointer is determined by summing up the movement vectors of all participating physical pointing devices.

The SmartBoard designers have developed quite useful tools for application sharing and joint hypertext authoring. However, just providing cooperative services is not enough. A set of intuitive graphical user interface metaphors also has to be designed so that users of standard applications can easily take advantage of the added functionality. For example, to broadcast a window from his screen to another machine, a user could select the window with a rubber-band metaphor, and then drag it onto a target host which he chooses from a graphical representation of the room.

Because of this, we are working on a representation that will show the CCF in a simplified $2 \frac{1}{2}$-D form. It will serve as an easily understandable interface that represents the complete CCF as a single 'object', offering cooperative functionality as well as access to the extended periphery (SmartBoards, etc.). It will also be a simple entry point to the virtual learning environment, VLE (see below).

\subsection{VLE - Virtual Learning Environment}

While the above cooperation control application still places the CCF as an object 'in front of the user', the 'Virtual Learning Enviroment', VLE, aims at bridging the gap between existing teleteaching technology and recent advancements in audio-visual representation. With VLE, users will be placed into a 3-dimensional world where every participant has his own workplace, following either an office- or a classroom/desk metaphor. Different kinds and levels of conversation and collaboration can be used by 'picking up' a virtual tool, or by moving into someone else's virtual office, into a lecture room, a marketplace, etc. To this end, different metaphors will be composed and offered alternatively, such as a book, library, classroom, blackboard, cinema, marketplace, auditorium, and office.

Together with these metaphors, authors of didactic material receive a toolbox to integrate their knowledge (e.g., as video or text) into the learning enviroment. The design of this collection of metaphors will leverage off recent developments in the areas of game consoles, virtual realities, PDA/PIC user interfaces (e.g., Magic Cap), etc. One important aspect is to make use of state-of-the-art telecommunications technology, e.g., teleconferencing, within these metaphors.

Those metaphors make up a consistent tool to navigate through (video) data, and to browse and retrieve information. They make cooperation and video-conferencing transparent to the users. Currently, the most promising technological basis for this project is VRML (Bell, 1995).

\subsection{Telecooperation}

As the six-year project Nestor (Mühlhäuser, 1995) on cooperative multimedia authoring and learning has proven, the cooperative use of 'cooperation-unaware' software is often insufficient for effective cooperation. Today's CSCW efforts are too much devoted to providing special-purpose cooperative tools, whereas component-based programming for usage-specific, individualized, 'cooperationaware' software is not sufficiently addressed. In Nestor, we developed such a supporting environment and used it, e.g., to develop custom cooperative courseware. Further development of such tools is planned in the context of a European-Union funded project to be carried out in the CCF. 


\subsection{Mobility and workflow support}

In our research on mobile computing - a crucial technology for flexible cooperative work - we realized that mobility support can be augmented considerably in the context of workflow management in its broadest sense. In the context of a long-lived workflow, mobility support can draw from a lot of semantic knowledge. The path of a mobile user, for example, can be automatically determined in the context of his future work items, the data reconciliation of 'home' and 'mobile' data can be achieved depending on the data semantics (known from workflow descriptions), etc.

As part of the MCW (Mobile Community and Workflow) initiative headed by Digital Equipment Corp., the Telecooperation research group currently co-develops a mobility-augmented ODBC service, enabling truly mobile use of many common PC tools which adhere to the ODBC standard.

This 'mobile OBDC' service is to become part of a 'mobile workflow' tools suite which is intended to draw from the synergy of the two domains as described.

\subsection{AHA - Advanced Hypermedia Access}

One central aspect of learning as we see it will be that information is not kept locally like the traditional library, but accessed via a network, overcoming the manifold problems of up-to-dateness, storage capacity, and abolishing the need to carry around a certain device containing your data, to name just a few.

To convey this message, CCF users need to be supplied with instant, high-speed, and effortless access to Internet resources, which are currently available through the World-Wide Web.

However, to show that the Web in its current form is far from being the perfect networked electronic medium, we wish to make users aware of its shortcomings as well as of its advantages.

One design option to address and overcome those problems is to use a Hyper-G server and/or clients. Hyper-G servers offer a number of conceptual advantages (Andrews, 1995):

- the concept of document collections: directory-like structures of documents and other collections which can be arranged hierarchically;

- document clusters: possibly mixed-media sets of documents to be presented in parallel, or to contain different language versions of a single document (this structure is orthogonal to the collections hierarchy);

- guided tours: a third independent way to specify relations between, or paths through, a number of documents;

- separation of contents and link information: this allows users to create links even in remote documents they do not have write access to;

- bidirectional links: links are stored in both directions, enabling administrators of a site to automatically inform all other sites with pointers to a certain page when it has moved elsewhere;

- automatic indexing of all documents: this makes full-text search in all documents a standard feature of any Hyper-G site;

- automatic caching: each Hyper-G server automatically works as a Proxy, using an object ID system that prevents old versions from being accessed;

- downward compatibility to standard WWW servers: a package exists that automatically converts an existing WWW server into a Hyper-G server.

Even though Hyper-G currently still has its own markup language (HTF), it understands HTML as well, and a beta version already supports the HTML 3.0 standard. Hyper-G servers are available for 
Unix platforms only; however, this would not pose problems for us since we have included a Unix fileserver in our equipment.

Clients for Hyper-G systems are available for Unix, PC, and Macintosh platforms. Their primary advantage is that they can display multiple views of the relation between the current document and its neighbours in the hypertext net, minimizing the risk of getting lost in hyperspace. Moreover, they allow the user to create new links interactively. Nevertheless, Hyper-G servers can be accessed with standard Web browsers like Netscape as well, and the loss of functionality is marginal, while at the same time many other features (like convenient hotlist management) become available.

While we will possibly run a Hyper-G server in the CCF, our WWW browser will probably be a standard system like Netscape, since the Hyper-G consortium also focuses its development efforts on the server side.

Another currently emerging standard, Java (Arnold, 1996), also represents a promising extension of WWW functionality. In our AHA installation, we will explain its advantages in terms of distributed component software (which may well change radically the way software is being distributed in the near future), but we are also currently using it as a platform-independent development tool, e.g., for the CCF control application.

\subsection{Telepresence}

Computers should primarily be used to augment the real (office) world where necessary and beneficial. One of those areas is bringing people together for a meeting, regardless of physical distance. While video-conferencing packages are readily available today, we want to go further. Participants should not sit in front of their PCs, staring at their monitors, but sit together at a desk which is partly real, and partly virtually extended. The wall-mounted display shows an extension of the conference desk, around which the external participants are arranged by the telepresence system.

This environment may be able to fulfil the need of a more personal and natural environment for telepresence activities, as recommended by similar efforts like, for example, the GreenSpace project (Mandeville, 1995).

The electronic wall described above will play a central role in the Telepresense setup, with its unique support for close-up, narrow-angle views. It will allow us to provide the illusion of a mirrored conference table in which the 'virtual' mirrored part is in reality a remote conference room.

\subsection{The electronic book}

Both conventional, paper-based, and modern, electronic media have a number of clear advantages (Wellner, 1993): Electronic documents are quick to edit, copy, transmit, share, file and retrieve. They allow for keyword searching, spell-checking, instant calculations, etc. Paper documents, on the other hand, are still cheaper, universally accepted, portable, familiar, and easier to read because of higher resolutions. They allow tactile handling and can easily be annotated with a pencil (and you can wrap them around your breakfast).

Up to now, research has not succeeded in combining those two sets of characteristics. Nevertheless, this will be a necessary precondition for electronic books to become a serious replacement for traditional paper in all its fields of application. Our group is doing research in that direction, and we hope to establish some cooperation with hardware vendors, using the AEC as a testbed for innovative devices. 


\subsection{MMC/EI: Multimedia Collaboration over EuroISDN}

Featuring ATM broadband networks, the German BERKOM multimedia teleservices (MMTS) demonstrated the feasibility of multimedia mail (MMM) and multimedia collaboration (MMC - audio/video conferencing, application sharing, and sophisticated conference management) on highly heterogeneous platforms. MMC/EI will extend the usability of MMC via specific adaptions to the EuroISDN narrowband standard. We hope to be able to showcase those developments in the Teleme$\mathrm{dia} /$ Teleteaching floor.

\subsection{SEAM - Semantic and Electronic Assistance for Meetings}

Most electronic meeting assistance projects are still limited to telecooperation features like application sharing and telepointing, remote liveboard control, audio/video conferencing, and joint editing. The SEAM project at the Telecooperation group aims beyond this level of support: It is intended to 'know' about semantic elements of meetings, such as topics, schedules, presenters, experts, moderators and other roles, presentations and discussions, action items, etc. That way, it can support more useful ways of querying multimedia meeting recordings, e.g., answer the question, 'What did John talk about while he used the interactive board to comment Anne's report on last year's sales?' by accessing the respective audio recordings and video indices directly.

\section{DEMONSTRATIONAL AND CURRICULAR CONTENTS}

As a computer science research group, we cannot create all the necessary end-user applications and curricular contents ourselves. Finding those components, however, is a task on its own that is not to be underestimated. The AEC is member of a number of EU projects (DEMOS, Employ, and others) that address those issues.

This, as well as cooperations with schools, adult education institutions, companies, and researchers all over the world, offers valuable contact to application and content providing partners who are in turn interested in the technical possibilities the infrastructure the AEC and esp. the Telemedia/Teleteaching floor have to offer. Describing that various sub-projects we are currently managing in this direction would, however, be outside the scope of this paper.

\section{CONCLUSION}

This paper presented our Class/Conference of the Future (CCF) project, an interdisciplinary effort to create an environment for cooperative working and learning, two activities which we identified as becoming more and more similar. After a summary of our conceptual goals for the project - to convey that learning will become a more situated, associative, and motivating experience - we described our solution strategies in bottom-up order.

Furniture considerations showed the necessity of reconfigurable desk setups, while hardware and operating system choices emphasized the importance of having different platforms available, with a focus on industry standards. Software infrastructure proved to be the major gap in cooperative systems today, and we described our own research projects in this area, while providing curricular content turned out to be a task that should be accomplished in close cooperation with external providers and institutions. 
In all, the conflicts between our CCF visions and practical considerations often forced us to accept compromises in our design, which is nevertheless an important experience for anybody who wishes to bridge the gap that still exists on our way to a real-world, usable international office of the future.

\subsection{Inventory recommendations}

For those who are confronted with the same problem as we were, to equip a learning and/or conference room using innovative concepts and technology, the following list may be helpful. It is divided into furniture, hardware, operating system, software infrastructure, and application requirements.

This list is by no means complete or compulsory, but rather a suggestion. Actual equipment decisions depend not only on what you plan to do with your room, but also on alliances with hardware providers and other companies: Your mileage may vary.

Table 1 Recommended equipment list for CCF-like projects

\begin{aligned} & \hline Pcs. Item \\ & \hline 16 configurable computer desks for 2 persons each \\ & \hline 1 Unix / Windows NT server \\ & 16 Pentium Multimedia PCs \\ & 6 Portable notebooks or similar devices \\ & 16 cameras \\ & 16 video boards \\ & 1 wall-mounted display \\ & 2 SMART Boards \\ & 2 active ISDN boards for video-conferencing \\ & 1 color laser printer \\ & 1 b/w laser printer \\ & 1 color scanner \\ & power and Ethernet/FDDI/ATM) network connections \\ & \hline 1 Unix or Windows NT server licence \\ & 16 Microsoft Windows 3.11, '95, and NT client licences \\ & \hline 1 process control application (running on server) \\ & 1 Hyper-G or WWW server licence \\ & 16 Intel ProShare licences + Smartboard driver \\ & 16 application sharing software licences \\ & \hline 16 Microsoft Office licences \\ & 16 Netscape licences \\ & additional 'contents' and demonstration applications and data \\ & \hline\end{aligned}




\section{REFERENCES}

Andrews, K., Kappe, F. and Maurer, H. (1995) Serving information to the Web with Hyper-G. Computer Networks and ISDN Systems, 27, 919-926.

Arnold, K. and Gosling, J. (1996) The Java Programming Language. Addison-Wesley, Reading.

Bell, G., Parisi, A. and Pesce, M. (1995) The Virtual Reality Modeling Language - Version 1.0 Specification (clarified), November 1995 (http://www.oki.com/vrml/vrml10c.html)

Hayter, M. (1993) Some computer science issues in Ubiquitous Computing. Commun. ACM, 36, 7, $75-84$.

Kay, A. (1991) Computers, networks, and education. Scientific American, Sept. 1991, 138-148.

Mandeville, J., Furness, T., Kawahata, M., Campbell, D., Danset, P., Dahl, A., Dauner, J., Davidson, J., Kandie, K. and Schwartz, P. (1995) GreenSpace: Creating a Distributed Virtual Environment for Global Applications. Proc. IEEE Networked Virtual Reality Workshop, Boston.

Mühlhäuser, M. (Ed.) (1995) Cooperative Computer-Aided Authoring and Learning. Kluwer Academic Publishers, Boston.

Shneiderman, B. (1995) Windows of Opportunity in Electronic Classrooms. Commun. ACM, 38, 11, 19-24.

Weiser, M. (1991) The Computer for the 21st Century. Scientific American, Sept. 1991, 66-75.

Wellner, P. (1993) Interacting with paper on the Digital Desk. Commun. ACM, 36, 7, 87-96.

\section{BIOGRAPHY}

Max Mühlhäuser is a Full Professor of Computer Science at Linz University, Austria, and head of the Telecooperation Research Group. After receiving his Doctorate in computer science from the University of Karlsruhe, Germany, in 1986, he worked as researcher there and at the Digital Equipment CASE Engineering Group in France, and set up and managed the CEC, a Digital Equipment research facility in Karlsruhe. After several years as professor in computer science at the Universities of Kaiserslautern and Karlsruhe, he visited the Eurecom Institute in Sophia Antipolis, France, in 1994, and has since accepted his new position at Linz University.

His research domain is software engineering for multimedia and multimodal interfaces, mobile and ubiquitous computing, human-human and human-computer cooperation. Applications include telelearning and telepresence, using distributed object-oriented and distributed hypermedia models as engineering approaches. He has published more than 70 articles, co-authored and edited books about distributed software engineering and computer-aided authoring/learning, and is a member of ACM, GI, and IEEE.

Jan Borchers studied computer science in Karlsruhe and London, and received his Master's Degree from the University of Karlsruhe in 1995. He now works on his Ph.D. at the Telecooperation Research Group, and is interested in user interfaces for new media and computer-aided learning, esp. in music education. He is the author of several papers about layout rules for electronic media, has written an interactive book about OSF/Motif, and is a member of ACM.

Chris Falkowski studied computer science and communications engineering in Aachen, and received his Master's Degree in 1989. After working as a software engineer for a large German software company for six years, he now works on his Ph.D. at the Telecooperation Research Group. His research interests focus on designing cooperative user environments.

Knut Manske studied computer science in Karlsruhe, where he received his Master's Degree in 1994. He now works on his Ph.D. at the Telecooperation Research Group, and is interested in video integration into multimedia systems, and multimodal user interfaces. 